\title{
Structural neuroimaging in the detection and prognosis of pre-clinical and early AD
}

\author{
Christine Fennema-Notestine ${ }^{\mathrm{a}, \mathrm{b}, *}$, Linda K. McEvoy ${ }^{\mathrm{b}}$, Donald J. Hagler, Jr. ${ }^{\mathrm{b}}$, Mark W. Jacobson ${ }^{\mathrm{a}, \mathrm{c}}$, \\ Anders M. Dale ${ }^{\mathrm{b}, \mathrm{d}}$ and the Alzheimer's Disease Neuroimaging Initiative ${ }^{1}$ \\ ${ }^{a}$ Deptartment of Psychiatry, University of California San Diego, San Diego, CA, USA \\ ${ }^{\mathrm{b}}$ Department of Radiology, University of California San Diego, San Diego, CA, USA \\ ${ }^{\mathrm{c}}$ Veterans Affairs San Diego Healthcare System, San Diego, CA, USA \\ ${ }^{\mathrm{d}}$ Department of Neurosciences, University of California San Diego, San Diego, CA, USA
}

\begin{abstract}
Current research supports the strong potential of structural MRI profiles, even within cross-sectional designs, as a promising method for the discrimination of Alzheimer's Disease (AD) from normal controls and for the prediction of Mild Cognitive Impairment (MCI) progression and conversion to AD. Findings suggest that measures of structural change in mesial and lateral temporal, cingulate, parietal and midfrontal areas may facilitate the assessment of a treatment's ability to halt the progressive structural loss that accompanies clinical decline in MCI. The performance of prediction is likely to continue to improve with the incorporation of measures from other neuroimaging modalities, clinical assessments, and neuromedical biomarkers, as the regional profile of individuals at risk for progression is refined.
\end{abstract}

Keywords: MRI, Alzheimer's disease, Mild Cognitive Impairment (MCI), morphometry, brain imaging

\section{Introduction}

As one of the most serious health issues facing the U.S. today, AD may afflict an estimated 13.2 million people in 2050, with current cost of caring for individuals with $\mathrm{AD}$ estimated at $\$ 148$ billion per year in the US [1,12]. Millions more individuals are suffering prodromal cognitive impairment that may be related to $\mathrm{AD}$ [40]. To facilitate the development of therapies aimed at preventing or delaying the progression of $\mathrm{AD}$, research has focused on the search for sensitive, non-

* Corresponding author: Christine Fennema-Notestine, Ph.D., UCSD School of Medicine, 9500 Gilman Dr \#0841, La Jolla, CA 92093-0841, USA. Tel.: +1 858822 3198; Fax: +1 858534 1078; E-mail: Fennema@UCSD.edu.

${ }^{1}$ This review emphasizes findings based on data obtained from the Alzheimer's Disease Neuroimaging Initiative (ADNI) database (www.loni.ucla.edu $\backslash \mathrm{ADNI}$ ). As such, the investigators within the ADNI contributed to the design and implementation of ADNI and/or provided data but did not participate in analysis or writing of this review. Complete listing of ADNI investigators available at http:// www.loni.ucla.edu/ADNI/Data/ADNI_Authorship_List.pdf. invasive, in vivo biomarkers that would enable earlier, more accurate clinical diagnosis and aid in monitoring disease progression and the effectiveness of therapeutic intervention $[26,35,36,82]$. Neuroimaging measures, which are sensitive to AD-related neurodegeneration early in the disease, hold significant promise as direct measures of disease-modifying treatment effects $[66,73,82]$. Numerous neuroimaging approaches provide insight into the neuronal and synaptic loss of $\mathrm{AD}$, including structural MRI, positron emission tomography (PET), and functional MRI. Such measures may improve the sensitivity and specificity of detecting $\mathrm{AD}$ at early stages and assist in identifying individuals at risk for developing AD. Early identification would allow treatment to begin before significant functional impairment or extensive irreversible neuronal damage occurs and may permit shorter treatment trials by focusing on patients with high likelihood of imminent conversion. Structural MRI measures in particular are non-invasive and relatively free from confounding behavioral factors, and, as a result, these measures may be less variable in assessing treatment effects relative 
to performance on commonly used cognitive tests [46, 48]. This decrease in variability may result in greater statistical power in clinical trials, enabling the use of smaller sample sizes. Cross-sectional, structural MRI research has provided insight into the neuroanatomical profiles of pre-clinical and early AD and has demonstrated significant value in the prediction of conversion and disease progression.

\section{Neuropathology of Alzheimer's disease}

Neuropathological studies have defined the basic neurodegenerative profile of AD through the distribution of hallmark features, amyloid plaques and neurofibrillary tangles. While amyloid deposits in the cerebral cortex show considerable inter-individual variation in density and distribution in the early stages of AD, the characteristic distribution of neurofibrillary pathology allows post-mortem differentiation of progressive stages of AD [9,10]. Neurofibrillary changes first appear in the transentorhinal area, then migrate into limbic areas before spreading into neocortical association areas $[9,10,66]$. Clinical diagnosis of AD typically accompanies the third stage, when modest neuropathology is observed in hippocampal structures and severe neuropathology is observed in the transentorhinal and entorhinal areas, affecting many of the projection neurons within these areas $[9,10]$. Structural MRI has provided insight into identifying the profile of early stage pathogenesis in vivo.

\section{Mild cognitive impairment}

The study of individuals with Mild Cognitive Impairment (MCI), which has been recognized as a potential prodromal stage of $\mathrm{AD}$, may increase our ability to detect early changes. MCI refers to a syndrome of impairment on one or more standardized tests of cognitive function of insufficient severity to cause functional impairment $[64,66,68,69]$. When memory is one of the cognitive domains involved, often referred to as "amnestic MCI," individuals are at increased risk of converting to $\mathrm{AD}$, estimated at $10-15 \%$ per year compared with $1-2 \%$ per year for cognitively intact elderly [68]. In addition, at autopsy the majority of amnestic MCI individuals show pathological features of $\mathrm{AD}$, supporting amnestic MCI as a transitional state between healthy aging and $\mathrm{AD}[60,64,70]$. MCI is a heterogeneous disorder, however, as some individuals do not progress, others convert to non-AD dementia, and a few revert to normal cognitive status [8]. Autopsy studies of amnestic MCI cases have confirmed this heterogeneity with individual cases demonstrating nonAD pathology, such as hippocampal sclerosis or subcortical ischemic vascular disease, despite similar cognitive characterization $[49,70]$. There is significant potential for neuroimaging to provide a more comprehensive characterization of AD-related structural changes in MCI individuals that may further define the prodromal stages of $\mathrm{AD}$.

\section{Cross-sectional structural MRI in AD and MCI}

As expected from the neuropathological and clinical profiles of AD, structural MRI has supported significant atrophy in mesial temporal structures affected early in the neurodegenerative process as well as in temporoparietal association areas $[3,9,10,71,77,83,84]$. These studies in AD individuals have also shown that early atrophy in these regions is followed by more extensive involvement of frontal and parietal regions, leaving primary and secondary sensory areas relatively spared until late in the disease $[3,9,10,77,83,84]$. Consequently, studies in early $\mathrm{AD}$ and in those at risk for $\mathrm{AD}$, such as individuals with MCI, have focused primarily on mesial temporal regions, demonstrating that hippocampal and entorhinal cortex typically are smaller than those measured in controls $[3,5,7,14,24,45,52,53,58,76,88]$, predictive of future conversion to $\mathrm{AD}[18,19,21,44,47,58$, $59,79]$, and correlated with memory impairment [25, 37]. The definitive classification and specificity of these changes to $\mathrm{AD}$, particularly for the hippocampus, remain a challenge [85], and ongoing work continues to examine additional regions and patterns of regional changes $[42,54,56,75,76,84,87]$. The cingulate cortex, for example, has been shown to exhibit changes in atrisk individuals $[41,50]$ and in those known to eventually convert to AD [57], and recent work suggests that MCI individuals with impairment in other cognitive domains in addition to memory (multiple-domain MCI, or MMCI) may evidence thinner precuneus cortices relative to individuals with impairments restricted to the memory domain (single domain MCI, or SMCI) [75]. An accurate depiction of the earliest patterns of degenerative changes will require extensive studies of individuals in both preclinical and prodromal stages of AD. 


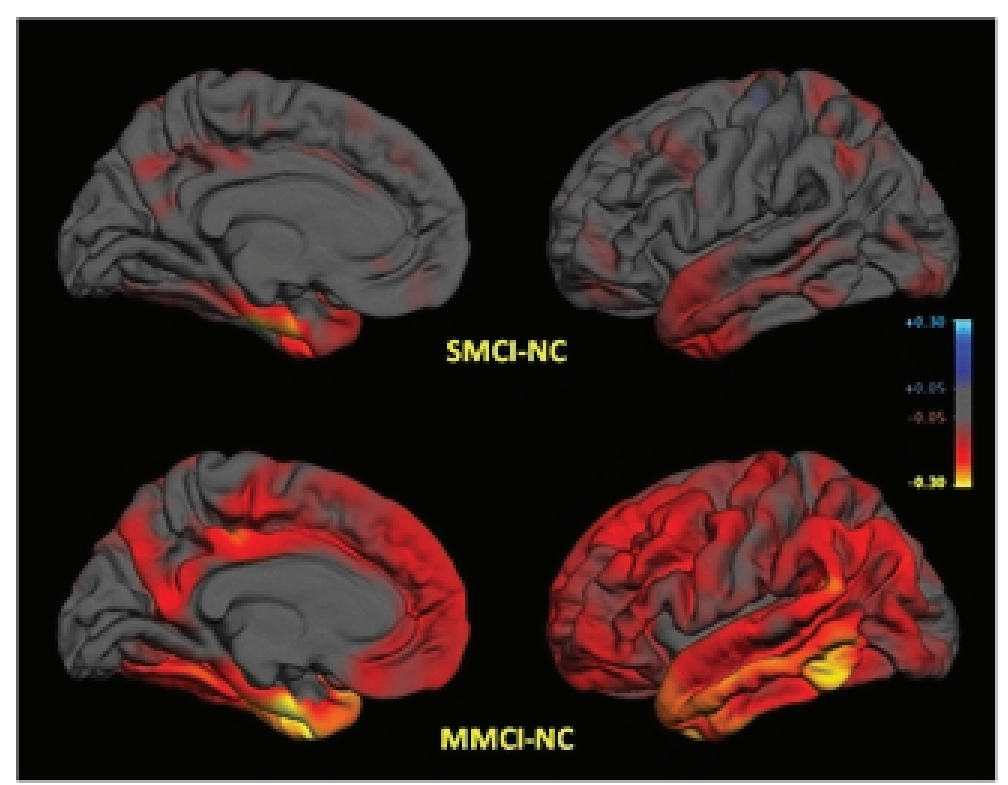

Fig. 1. Group differences in average thickness (mm) for left hemisphere. Top row: SMCI vs. NC; Bottom row: MMCI vs. NC. LEFT mesial views, RIGHT lateral views. The scale ranges from $<-0.3$ (yellow) to $>+0.3$ (cyan) mm thickness. Areas on the red-yellow spectrum indicate regions of thinning with disease: approximate color scale in mm is -0.05 to -0.15 dark red, -0.20 bright red, -0.25 orange, and $<-0.30$ yellow. Any differences smaller than $+/-0.05 \mathrm{~mm}$ are gray.

\section{Neuroanatomical profiles}

In this context, our recent work has emphasized the role of cross-sectional, structural MRI in providing neuroanatomical profiles of pre-clinical and early $\mathrm{AD}$ and in the prediction of disease progression [29, 61]. Our studies are based on data publicly available from the multi-site Alzheimer's Disease Neuroimaging Initiative (ADNI), an important effort designed to facilitate the scientific evaluation of neuroimaging and other biomarkers in the onset and progression of MCI and $\mathrm{AD}[43,65,66]$. Using methods developed within the NIH/NCRR sponsored Morphometry Biomedical Informatics Research Network (mBIRN) and the ADNI [30,31,33,39,43,51], we studied normal elderly participants and individuals with MCI and AD. The MCI participants were impaired on one or more standardized tests of cognitive function, with memory as one of the impaired domains (i.e., amnestic MCI); demonstrated no associated functional impairment; and did not meet criteria for clinical diagnosis of dementia at the time of these baseline scans [68]. In addition, during our morphometric characterization, we defined neuropsychological subgroups of the MCI cohort, dividing the group into those with SMCI, which may reflect the earliest stage of prodromal AD, and those with MMCI, which may represent a later disease state.

\section{Characterization of single-domain, amnestic MCI}

We characterized the MCI $(n=175)$ and $\operatorname{AD}(n=$ $84)$ cohorts relative to the normal controls $(n=139)$ using methods based on volumetric segmentation [30, 31] and cortical surface reconstruction and parcellation $[15,16,20,32,33]$ techniques for each individual's brain, combining region of interest (ROI) and cortical surface vertex-wide approaches that allow greater exploitation of information obtained from all brain areas [29]. The results demonstrated robust performance of the methods and evidenced measurements sufficiently sensitive to reveal the subtle morphometric characterization of SMCI and MMCI in this cross-sectional sample of individuals who may be in a prodromal AD state (Fig. 1). While significant mesial temporal atrophy characterized all groups, including SMCI, MM$\mathrm{CI}$, and $\mathrm{AD}$, smaller volumes and thinner cortex were widespread and evident across the cortex even within the SMCI group.

The regions that were most sensitive to early changes were within temporal, rostral posterior cingulate, inferior parietal, precuneus, and caudal midfrontal cortices (Fig. 1). Areas critical for distinguishing SMCI from $\mathrm{NC}$ were in the mesial temporal regions as expected, including bilateral hippocampus, bilateral entorhinal cor- 

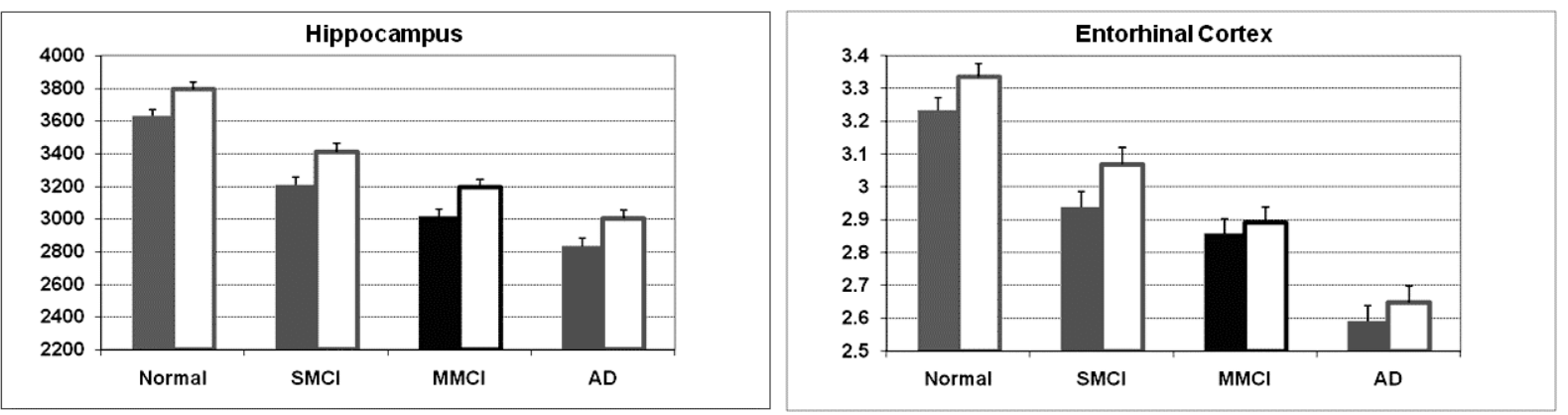

Fig. 2. Estimated marginal mean volume (mm3) for Hippocampus and Entorhinal Cortex thickness (mm) by group accounting for sex and age effects. Error bars $=$ std error of the mean. Solid bar = Left Hemisphere; outlined open bar = Right Hemisphere.

tex, and left amygdala, similar to recent findings [42, $75,76,87]$, supporting these regions as the most sensitive to early AD-related changes. Given previous work suggesting that mesial temporal asymmetries also may be modified by disease state, we also examined left/right asymmetries as potential measures indicative of risk or disease progression. Building on findings in AD (e.g. [4,55,83]), several investigators [75,78] have suggested that left hemisphere regions may be more affected than the right, changing the natural asymmetries, in individuals at increased risk for $\mathrm{AD}$, although reports are conflicting [56]. Our findings supported natural right dominant asymmetries in the hippocampus and entorhinal cortex of normal controls, as suggested in some previous work (e.g. [28,67]), however, these asymmetries did not interact with diagnostic group providing no support for structural asymmetries as an indication of disease state (Fig. 2).

Importantly, in the SMCI cohort, characterized with impairment in the memory domain only, significant atrophy extended beyond the mesial temporal regions into posterior cingulate, parietal, and frontal regions indicating that widespread damage occurs before standard clinical measures can detect AD. The pattern of thinning in SMCI overlapped to some extent with recent findings by Seo and colleagues [75], although our larger sample demonstrated greater thinning in medial parietal, lateral middle temporal, and anterior cingulate regions and powered significant differences from $\mathrm{NC}$ in numerous ROIs. These broad changes even within individuals impaired only in the memory domain suggest that standard neuropsychological measures may not be sufficiently sensitive, although novel approaches may be more informative (see Jacobson et al., this issue). The pattern of results also broadly supported the view that MMCI may be a later stage of prodromal AD than SMCI, although some of the structural changes observed confirmed the potential heterogene- ity of the sample. Relative to SMCI, the presumably later stage of MMCI showed greater atrophy in the inferior and lateral temporal lobe, and significantly thinner temporo-parietal association cortices, retrosplenial, anterior cingulate, and other frontal regions; these significant findings were more widespread relative to previous work [75], perhaps due to a larger MMCI sample. The AD and MMCI groups were relatively similar, with greater differences in some parietal, retrosplenial and frontal regions. These profiles may prove useful in defining a pattern of degeneration that will enable discrimination of early stage AD from other disorders that also impact mesial temporal regions.

\section{Proposed trajectory based on cross-sectional data}

In this cross-sectional sample, we further explored the potential "progression" from NC to AD employing regression models assessing the linear and quadratic effects. As expected, the earliest and most dramatic effects across groups were evident within the entorhinal cortex. Rostral posterior cingulate was reduced, similarly across groups, followed by additional temporal, parietal, and frontal regions. Of the later changing regions, the lateral middle temporal gyrus may change most rapidly, followed by the posterior cingulate and inferior parietal cortices, and then the rostral middle frontal region. The proposed sequence of change supports neuropathological findings as degeneration begins in the mesial temporal area, moves through rostral posterior cingulate and medial orbitofrontal cortices, followed by lateral temporal, retrosplenial and inferior parietal regions, and, subsequently, mid frontal cortex. The growing body of evidence thus suggests that there is significant, widespread cortical atrophy in preclinical stages of $\mathrm{AD}$, including areas outside the mesial 
temporal lobe. These cross-sectional findings then lead to the question of whether changes in these regions are predictive of conversion to AD.

\section{Discrimination, classification, and prediction}

Methods to facilitate the prediction of which MCI individuals are likely to convert to AD could increase efficiency of clinical treatment trials by allowing them to focus on individuals with high likelihood of imminent conversion, permitting shorter follow-up periods to demonstrate efficacy, alongside a potential utility for individual clinical prognosis. While previous work has supported the predictive power of mesial temporal regions for future conversion to $\mathrm{AD}[2,18,19,21$, $23,44,47,58,59,79]$, these more extensive morphometric findings in MCI may increase the power to predict conversion to AD. Cross-sectional differences in hippocampal and entorhinal volumes have been found to discriminate between MCI patients who convert to $\mathrm{AD}$ and those who remain stable $[21,47,58,79]$. Crosssectional differences in other brain areas, such as the amygdala [19] or the volume of the temporal horn of the lateral ventricle [59], have also been shown to predict conversion to AD. However, these classifiers may have limited specificity to AD [47].

In this light, combinations of regional structural measures may increase accuracy in predicting imminent risk of conversion to $\mathrm{AD}$. Our recent work [61] employed multivariate procedures to capitalize on this morphometric signature associated with early $\mathrm{AD}$ in the prediction of disease progression in MCI, potentially increasing both specificity and sensitivity in detecting $\mathrm{AD}$ at early stages. Several studies have shown improvement in classification accuracy when more than one measure is used, often combining hippocampus and an additional measure such as the amygdala or posterior cingulate volumes $[11,38,47,80]$. Additional small studies have approached the search for a predictive structural profile through voxel-based techniques, such as density maps and voxel based morphometry [17, 81]. In a study of MCI and control individuals, a highdimensional pattern classification technique resulted in a cross-validated accuracy of $90 \%$ for discriminating $15 \mathrm{MCI}$ from 15 matched controls [17]. The resulting classification algorithm included areas within the lateral and inferior regions of the hippocampus, and regions in bilateral superior, middle, and inferior temporal gyri, bilateral orbitofrontal, left fusiform and posterior cingulate. Another study found that although the voxel- based approach resulted in superior classification relative to hippocampal volume alone, the combination of the two provided the best classification [81]. These studies demonstrate the promise of employing patterns of structural information in classification, although the reliability of these approaches in larger samples with individuals further from conversion to $\mathrm{AD}$ should be examined. The potential for neuroimaging biomarkers to be predictive of conversion risk based on a baseline assessment for individuals with MCI may prove to be more advantageous.

This issue is presently under investigation by several groups using baseline data from the ADNI. Fan and colleagues [27] analyzed baseline MRI data from 66 controls, 88 individuals with MCI, and 56 individuals with AD from the ADNI. High dimensional pattern classification methods were applied to voxel based morphometric measures from the healthy controls and AD subjects to identify a pattern of atrophy characteristic of AD. The resulting pattern was spatially complex, involving widespread brain regions. Importantly, individuals with MCI who displayed this pattern showed greater 1-year clinical decline than those who did not [27].

\section{Discrimination of controls and AD}

We have recently extended these findings by applying multivariate procedures to a larger subset of the ADNI cohort (the ADNI sample characterized earlier [29], which included 139 controls, 175 individuals with MCI, and 84 with AD) [61]. We were able to identify a pattern of regional atrophy characteristic of $\mathrm{AD}$ and to demonstrate that the presence of this pattern in individuals with MCI was predictive of 1-year clinical decline and structural volume loss [61]. With stepwise linear discriminant analysis (LDA) using candidate input morphometric variables from 58 ROIs, including lateral ventricles, mesial temporal structures, and cortical association areas, we identified a pattern of regional atrophy that best discriminated AD from NC. The regional pattern that best discriminated NC from AD subjects involved eight measures, including left hippocampal volume and cortical thickness of right entorhinal, right middle temporal, left bank of the superior temporal sulcus, right retrosplenial, right superior temporal, left medial orbital frontal, and right lateral orbital frontal regions. 


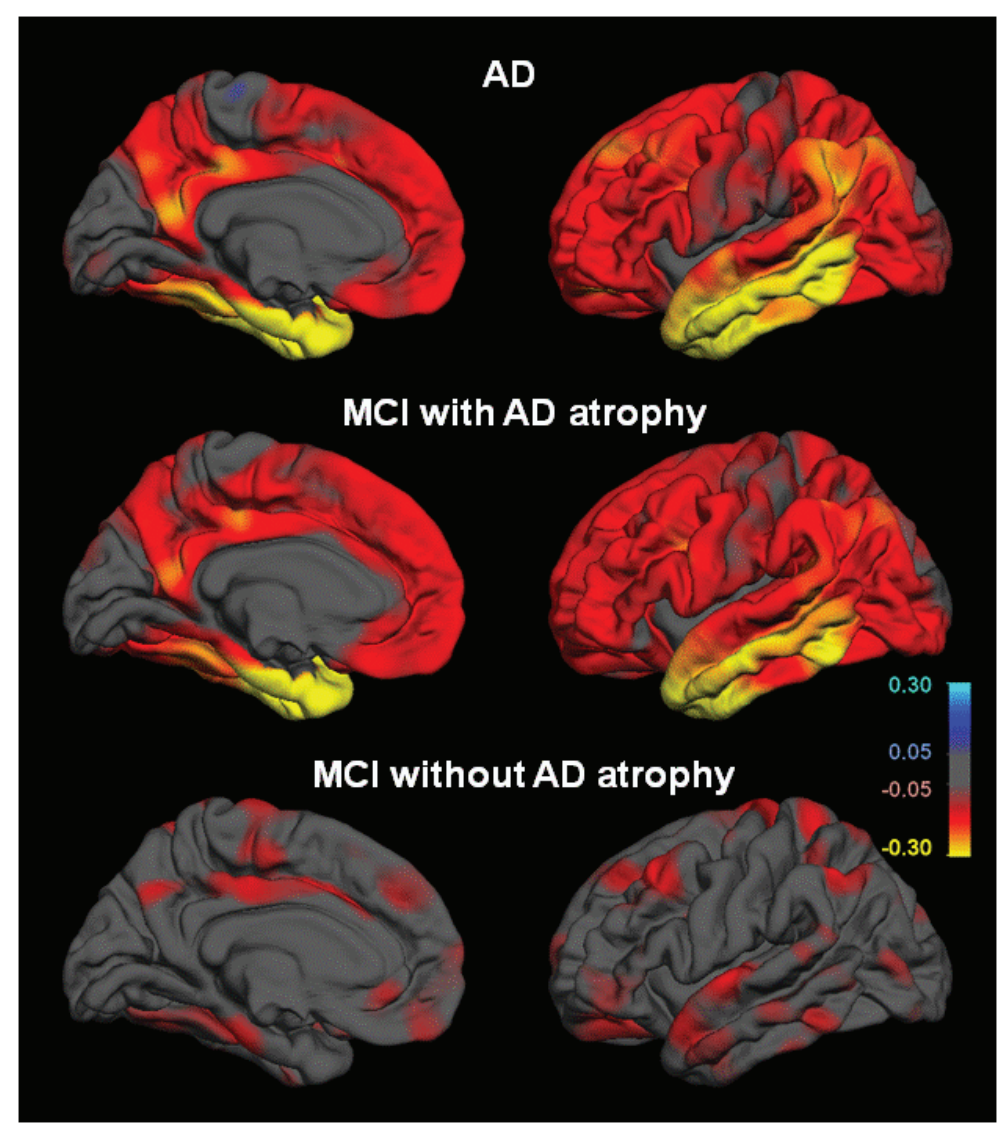

Fig. 3. Group differences in average thickness (mm) for left hemisphere. All comparisons are relative to NC cohort. Top row: AD vs. NC; Middle: MCI subjects showing AD atrophy pattern vs. NC; Bottom: MCI subjects without AD atrophy pattern vs. NC. LEFT mesial views, RIGHT lateral views. The scale ranges from $<-0.3$ (yellow) to $>+0.3$ (cyan) $\mathrm{mm}$ thickness. Areas on the red-yellow spectrum indicate regions of thinning with disease.

\section{Classification of MCI subgroups}

This discriminant model was then applied to MCI data, producing an "atrophy score" for each MCI individual reflecting the degree to which the individual's MRI reflected the presence of characteristic AD atrophy. On the basis of this score, each MCI participant was classified as showing either the NC or AD "imaging phenotype" (Fig. 3). MCI participants with $\mathrm{AD}$ atrophy showed an imaging pattern nearly identical to that of the AD group, with structural thinning evident even in regions not contributing to the atrophy score. MCI subjects without the AD atrophy pattern displayed less widespread atrophy, with a reduced level of atrophy in hippocampal and middle temporal regions. MCI individuals with AD atrophy had higher baseline CDR sum of boxes and lower verbal memory scores but equivalent MMSE scores at baseline.

In essence, the pattern that best discriminated $\mathrm{NC}$ from $\mathrm{AD}$, and that was predictive of decline in MCI, in- volved the mesial temporal, lateral temporal, retrosplenial and orbitofrontal regions. Mesial temporal structures have long been implicated early in AD, and atrophy in these structures has been found to be predictive of disease progression [21,22,44,58,79]. Lateral temporal areas, including inferior, middle, and superior temporal gyri, have been implicated in the progression of AD [13,72,86,87], and we demonstrated in our morphometric study that there is also evidence of such changes in SMCI [29]. These findings support a pattern of preclinical cortical atrophy that may be predictive of a more rapid course of disease progression.

\section{Prediction of clinical decline}

Of significant interest, follow-up data were available for 160 of these MCI cases for a year from baseline evaluation. Those expressing the baseline AD phe- 
notype showed a significant decline in MMSE score over one year, whereas those with the NC phenotype remained stable. The atrophy score was the primary predictor for this decline, although additional power in prediction came from Apolipoprotein E (APOE) $\varepsilon 4$ allele status (a genetic risk factor for late-onset AD [6]) and Logical Memory Delayed Recall scores. Interestingly, four MCI individuals reverted to normal cognitive status (three showed the NC imaging phenotype) and 33 converted to a diagnosis of AD (23 showed the AD phenotype). Those who declined in MMSE score over one year were significantly more likely to show the AD phenotype, the conversion rate among MCI subjects with the $\mathrm{AD}$ phenotype was $32 \%$ relative to only $11 \%$ for those with the NC phenotype. This contrasts with a recent report that indicated that whole brain, ventricular, entorhinal and hippocampal volumes did not provide additional predictive information of clinical decline beyond that attainable with clinical measures [34], and shows the value of examining individually specific brain regions beyond the mesial temporal lobe.

\section{Summary}

In summary, the literature supports the strong potential of structural MRI profiles, even within crosssectional designs, as a promising method for the discrimination of $\mathrm{AD}$ from normal controls and in the prediction of MCI progression and conversion to AD. Findings suggest that measures of structural change in mesial and lateral temporal, cingulate, parietal and midfrontal areas may facilitate the assessment of a treatment's ability to halt the progressive structural loss that accompanies clinical decline in MCI. Of course, there remains significant potential to improve the performance of prediction. For example, using follow-up data from ADNI's large MCI cohort to identify structural patterns associated with MCI individuals who convert to AD relative to those who do not [62] may better identify the structural changes that are predictive of disease progression. In addition, the incorporation of measures from other neuroimaging modalities, clinical assessments, and neuromedical biomarkers, as being assessed in current work (e.g. [63,74]), may also help to refine the regional profile of individuals at risk for progression.

\section{Acknowledgments}

This work was supported by grants from the National Center for Research Resources (U24 RR021382 CFN, AMD; DJH); the National Institute on Aging (R01AG22381 - CFN, LKM, AMD; K01AG029218 LKM); and the Department of Veterans Affairs (Advanced Career Dev. Award - MWJ). This review refers to important work facilitated by the Alzheimer's Disease Neuroimaging Initiative (ADNI; Principal Investigator: Michael Weiner; NIH grant U01 AG024904), which funded data collection and sharing. ADNI is funded by the National Institute on Aging, the National Institute of Biomedical Imaging and Bioengineering (NIBIB), and through generous contributions from the following: Pfizer Inc., Wyeth Research, Bristol-Myers Squibb, Eli Lilly and Company, GlaxoSmithKline, Merck \& Co. Inc., AstraZeneca AB, Novartis Pharmaceuticals Corporation, Alzheimer's Association, Eisai Global Clinical Development, Elan Corporation plc, Forest Laboratories, and the Institute for the Study of Aging, with participation from the U.S. Food and Drug Administration. Industry partnerships are coordinated through the Foundation for the National Institutes of Health. The grantee organization is the Northern California Institute for Research and Education, and the study is coordinated by the Alzheimer's Disease Cooperative Study at the University of California, San Diego. ADNI data are disseminated by the Laboratory of Neuro Imaging at the University of California, Los Angeles.

\section{References}

[1] Alzheimer's Association, 2009 Alzheimer's Disease Facts and Figures, Alzheimers Dement 5 (2009).

[2] L.G. Apostolova, R.A. Dutton, I.D. Dinov, K.M. Hayashi, A.W. Toga, J.L. Cummings and P.M. Thompson, Conversion of mild cognitive impairment to Alzheimer disease predicted by hippocampal atrophy maps, Arch Neurol 63 (2006), 693699.

[3] M. Atiya, B.T. Hyman, M.S. Albert and R. Killiany, Structural magnetic resonance imaging in established and prodromal Alzheimer disease: a review, Alzheimer Dis Assoc Disord 17 (2003), 177-195.

[4] J. Barnes, R.I. Scahill, J.M. Schott, C. Frost, M.N. Rossor and N.C. Fox, Does Alzheimer's disease affect hippocampal asymmetry? Evidence from a cross-sectional and longitudinal volumetric MRI study, Dement Geriatr Cogn Disord 19 (2005), 338-344.

[5] S. Bell-McGinty, O.L. Lopez, C.C. Meltzer, J.M. Scanlon, E.M. Whyte, S.T. Dekosky and J.T. Becker, Differential cortical atrophy in subgroups of mild cognitive impairment, Arch Neurol 62 (2005), 1393-1397. 
[6] D. Blacker, J.L. Haines, L. Rodes, H. Terwedow, R.C. Go, L.E. Harrell, R.T. Perry, S.S. Bassett, G. Chase, D. Meyers et al., ApoE-4 and age at onset of Alzheimer's disease: the NIMH genetics initiative, Neurology 48 (1997), 139-147.

[7] M. Bobinski, M.J. de Leon, A. Convit, S. De Santi, J. Wegiel, C.Y. Tarshish, L.A. Saint Louis and H. M. Wisniewski, MRI of entorhinal cortex in mild Alzheimer's disease, Lancet 353 (1999), 38-40.

[8] P.A. Boyle, R.S. Wilson, N.T. Aggarwal, Y. Tang and D.A. Bennett, Mild cognitive impairment: risk of Alzheimer disease and rate of cognitive decline, Neurology 67 (2006), 441445.

[9] H. Braak, I. Alafuzoff, T. Arzberger, H. Kretzschmar and K. Del Tredici, Staging of Alzheimer disease-associated neurofibrillary pathology using paraffin sections and immunocytochemistry, Acta Neuropathol (Berl) 112 (2006), 389-404.

[10] H. Braak and E. Braak, Neuropathological stageing of Alzheimer-related changes, Acta Neuropathol (Berl) 82 (1991), 239-259.

[11] D.J. Callen, S.E. Black, F. Gao, C.B. Caldwell and J.P. Szalai, Beyond the hippocampus: MRI volumetry confirms widespread limbic atrophy in AD, Neurology 57 (2001), 16691674.

[12] Centers for Disease Control and Prevention National Center for Chronic Disease Prevention and Health Promotion, Unrealized prevention opportunities: Reducing the health and economic burden of chronic illness.

[13] A. Convit, J. de Asis, M.J. de Leon, C.Y. Tarshish, S. De Santi and H. Rusinek, Atrophy of the medial occipitotemporal, inferior, and middle temporal gyri in non-demented elderly predict decline to Alzheimer's disease, Neurobiol Aging 21 (2000), 19-26.

[14] A. Convit, M.J. De Leon, C. Tarshish, S. De Santi, W. Tsui, H. Rusinek and A. George, Specific hippocampal volume reductions in individuals at risk for Alzheimer's disease, Neurobiol Aging 18 (1997), 131-138.

[15] A.M. Dale, B. Fischl and M.I. Sereno, Cortical surface-based analysis. I. Segmentation and surface reconstruction, $\mathrm{Neu}$ rolmage 9 (1999), 179-194.

[16] A.M. Dale and M.I. Sereno, Improved localization of cortical activity by combining EEG and MEG with MRI cortical surface reconstruction: A linear approach, Journal of Cognitive Neuroscience 5 (1993), 162-176.

[17] C. Davatzikos, Y. Fan, X. Wu, D. Shen and S.M. Resnick, Detection of prodromal Alzheimer's disease via pattern classification of magnetic resonance imaging, Neurobiol Aging 29 (2008), 514-523.

[18] M.J. de Leon, S. DeSanti, R. Zinkowski, P.D. Mehta, D. Pratico, S. Segal, C. Clark, D. Kerkman, J. DeBernardis, J. Li et al., MRI and CSF studies in the early diagnosis of Alzheimer's disease, J Intern Med 256 (2004), 205-223.

[19] T. den Heijer, M.I. Geerlings, F.E. Hoebeek, A. Hofman, P.J. Koudstaal and M.M. Breteler, Use of hippocampal and amygdalar volumes on magnetic resonance imaging to predict dementia in cognitively intact elderly people, Arch Gen Psychiatry 63 (2006), 57-62.

[20] R.S. Desikan, F. Segonne, B. Fischl, B.T. Quinn, B.C. Dickerson, D. Blacker, R.L. Buckner, A.M. Dale, R.P. Maguire, B.T. Hyman et al., An automated labeling system for subdividing the human cerebral cortex on MRI scans into gyral based regions of interest, NeuroImage 31 (2006), 968-980.

[21] L. deToledo-Morrell, T.R. Stoub, M. Bulgakova, R.S. Wilson, D.A. Bennett, S. Leurgans, J. Wuu and D.A. Turner, MRI- derived entorhinal volume is a good predictor of conversion from MCI to AD, Neurobiol Aging 25 (2004), 1197-1203.

[22] D.P. Devanand, D.M. Jacobs, M.X. Tang, C. Del CastilloCastaneda, M. Sano, K. Marder, K. Bell, F.W. Bylsma, J. Brandt, M. Albert et al., The course of psychopathologic features in mild to moderate Alzheimer disease, Arch Gen Psychiatry 54 (1997), 257-263.

[23] D.P. Devanand, G. Pradhaban, X. Liu, A. Khandji, S. De Santi, S. Segal, H. Rusinek, G.H. Pelton, L.S. Honig, R. Mayeux et al., Hippocampal and entorhinal atrophy in mild cognitive impairment: prediction of Alzheimer disease, Neurology 68 (2007), 828-836.

[24] A.T. Du, N. Schuff, D. Amend, M.P. Laakso, Y.Y. Hsu, W.J. Jagust, K. Yaffe, J.H. Kramer, B. Reed, D. Norman et al., Magnetic resonance imaging of the entorhinal cortex and hippocampus in mild cognitive impairment and Alzheimer's disease, J Neurol Neurosurg Psychiatry 71 (2001), 441-447.

[25] A.T. Du, N. Schuff, J.H. Kramer, S. Ganzer, X.P. Zhu, W.J. Jagust, B.L. Miller, B.R. Reed, D. Mungas, K. Yaffe et al., Higher atrophy rate of entorhinal cortex than hippocampus in AD, Neurology 62 (2004), 422-427.

[26] B. Dubois, H.H. Feldman, C. Jacova, S.T. Dekosky, P. Barberger-Gateau, J. Cummings, A. Delacourte, D. Galasko, S. Gauthier, G. Jicha et al., Research criteria for the diagnosis of Alzheimer's disease: revising the NINCDS-ADRDA criteria, Lancet Neurol 6 (2007), 734-746.

[27] Y. Fan, N. Batmanghelich, C.M. Clark and C. Davatzikos, Spatial patterns of brain atrophy in MCI patients, identified via high-dimensional pattern classification, predict subsequent cognitive decline, NeuroImage 39 (2008), 1731-1743.

[28] C. Fennema-Notestine, A.C. Gamst, B.T. Quinn, J. Pacheco, T.L. Jernigan, L. Thal, R. Buckner, R. Killiany, D. Blacker, A.M. Dale et al., Feasibility of multi-site clinical structural neuroimaging studies of aging using legacy data, Neuroinformatics 5 (2007), 235-245.

[29] C. Fennema-Notestine, D.J. Hagler, Jr., L.K. McEvoy, A.S. Fleisher, E.H. Wu, D.S. Karow, A.M. Dale and the ADNI, Structural MRI biomarkers for preclinical and mild Alzheimer's Disease, Human Brain Mapping, (2009).

[30] B. Fischl, D.H. Salat, E. Busa, M. Albert, M. Dieterich, C. Haselgrove, A. van der Kouwe, R. Killiany, D. Kennedy, S. Klaveness et al., Whole brain segmentation: automated labeling of neuroanatomical structures in the human brain, Neuron 33 (2002), 341-355.

[31] B. Fischl, D.H. Salat, A.J. van der Kouwe, N. Makris, F. Segonne, B.T. Quinn and A.M. Dale, Sequence-independent segmentation of magnetic resonance images, NeuroImage 23(Suppl 1) (2004), S69-S84.

[32] B. Fischl, M.I. Sereno and A.M. Dale, Cortical surface-based analysis. II: Inflation, flattening, and a surface-based coordinate system, NeuroImage 9 (1999), 195-207.

[33] B. Fischl, A. Van Der Kouwe, C. Destrieux, E. Halgren, F. Segonne, D.H. Salat, E. Busa, L.J. Seidman, J. Goldstein and D. Kennedy et al., Automatically parcellating the human cerebral cortex, Cereb Cortex 14 (2004), 11-22.

[34] A.S. Fleisher, S. Sun, C. Taylor, C.P. Ward, A.C. Gamst, R.C. Petersen, C.R. Jack, Jr., P.S. Aisen and L.J. Thal, Volumetric MRI vs clinical predictors of Alzheimer disease in mild cognitive impairment, Neurology 70 (2008), 191-199.

[35] R.A. Frank, D. Galasko, H. Hampel, J. Hardy, M.J. de Leon, P.D. Mehta, J. Rogers, E. Siemers and J.Q. Trojanowski, Biological markers for therapeutic trials in Alzheimer's disease. Proceedings of the biological markers working group; NIA 
initiative on neuroimaging in Alzheimer's disease, Neurobiol Aging 24 (2003), 521-536.

[36] D. Galasko, Biomarkers for Alzheimer's disease - clinical needs and application, J Alzheimers Dis 8 (2005), 339-346.

[37] M. Grundman, C.R. Jack, Jr., R.C. Petersen, H.T. Kim, C. Taylor, M. Datvian, M.F. Weiner, C. DeCarli, S.T. DeKosky, C. van Dyck et al., Hippocampal volume is associated with memory but not nonmemory cognitive performance in patients with mild cognitive impairment, J Mol Neurosci 20 (2003), 241-248.

[38] H. Hampel, S.J. Teipel, W. Bayer, G.E. Alexander, R. Schwarz, M.B. Schapiro, S.I. Rapoport and H.J. Moller, Age transformation of combined hippocampus and amygdala volume improves diagnostic accuracy in Alzheimer's disease, J Neurol Sci 194 (2002), 15-19.

[39] X. Han, J. Jovicich, D. Salat, A. van der Kouwe, B. Quinn, S. Czanner, E. Busa, J. Pacheco, M. Albert, R. Killiany et al., Reliability of MRI-derived measurements of human cerebral cortical thickness: The effects of field strength, scanner upgrade and manufacturer, NeuroImage (2006).

[40] L.E. Hebert, P.A. Scherr, J.L. Bienias, D.A. Bennett and D.A. Evans, Alzheimer disease in the US population: prevalence estimates using the 2000 census, Arch Neurol 60 (2003), 1119_ 1122 .

[41] K. Hirao, T. Ohnishi, H. Matsuda, K. Nemoto, Y. Hirata, F. Yamashita, T. Asada and T. Iwamoto, Functional interactions between entorhinal cortex and posterior cingulate cortex at the very early stage of Alzheimer's disease using brain perfusion single-photon emission computed tomography, Nucl Med Commun 27 (2006), 151-156.

[42] X. Hua, A.D. Leow, N. Parikshak, S. Lee, M.C. Chiang, A.W. Toga, C.R. Jack, Jr., M.W. Weiner and P.M. Thompson, Tensor-based morphometry as a neuroimaging biomarker for Alzheimer's disease: an MRI study of $676 \mathrm{AD}, \mathrm{MCI}$, and normal subjects, NeuroImage 43 (2008), 458-469.

[43] C.R. Jack, Jr., M.A. Bernstein, N.C. Fox, P. Thompson, G. Alexander, D. Harvey, B. Borowski, P.J. Britson, L.W.J, C. Ward et al., The Alzheimer's Disease Neuroimaging Initiative (ADNI): MRI methods, J Magn Reson Imaging 27 (2008), 685-691.

[44] C.R. Jack, Jr., R.C. Petersen, Y.C. Xu, P. C. O’Brien, G.E. Smith, R.J. Ivnik, B.F. Boeve, S.C. Waring, E.G. Tangalos and E. Kokmen, Prediction of AD with MRI-based hippocampal volume in mild cognitive impairment, Neurology 52 (1999), 1397-1403.

[45] C.R. Jack, Jr., R.C. Petersen, Y.C. Xu, S.C. Waring, P.C. O'Brien, E.G. Tangalos, G.E. Smith, R.J. Ivnik and E. Kokmen, Medial temporal atrophy on MRI in normal aging and very mild Alzheimer's disease, Neurology 49 (1997), 786794.

[46] C.R. Jack, Jr., M.M. Shiung, J.L. Gunter, P.C. O'Brien, S.D. Weigand, D.S. Knopman, B.F. Boeve, R.J. Ivnik, G.E. Smith, R.H. Cha et al., Comparison of different MRI brain atrophy rate measures with clinical disease progression in $\mathrm{AD}, \mathrm{Neu}$ rology 62 (2004), 591-600.

[47] C.R. Jack, Jr., M.M. Shiung, S.D. Weigand, P.C. O’Brien, J.L. Gunter, B.F. Boeve, D.S. Knopman, G.E. Smith, R.J. Ivnik, E.G. Tangalos et al., Brain atrophy rates predict subsequent clinical conversion in normal elderly and amnestic MCI, Neurology $\mathbf{6 5}$ (2005), 1227-1231.

[48] C.R. Jack, Jr., M. Slomkowski, S. Gracon, T.M. Hoover, J.P. Felmlee, K. Stewart, Y. Xu, M. Shiung, P. C. O’Brien, R. Cha et al., MRI as a biomarker of disease progression in a therapeutic trial of milameline for AD, Neurology 60 (2003),
253-260.

[49] G.A. Jicha, R.C. Petersen, D.S. Knopman, B.F. Boeve, G.E. Smith, Y.E. Geda, K.A. Johnson, R. Cha, M.W. Delucia, H. Braak et al., Argyrophilic grain disease in demented subjects presenting initially with amnestic mild cognitive impairment, J Neuropathol Exp Neurol 65 (2006), 602-609.

[50] B.F. Jones, J. Barnes, H.B. Uylings, N.C. Fox, C. Frost, M.P. Witter and P. Scheltens, Differential Regional Atrophy of the Cingulate Gyrus in Alzheimer Disease: A Volumetric MRI Study, Cereb Cortex (2006).

[51] J. Jovicich, S. Czanner, D. Greve, E. Haley, A. van der Kouwe, R. Gollub, D. Kennedy, F. Schmitt, G. Brown, J. Macfall et al., Reliability in multi-site structural MRI studies: effects of gradient non-linearity correction on phantom and human data, NeuroImage 30 (2006), 436-443.

[52] K. Juottonen, M.P. Laakso, K. Partanen and H. Soininen, Comparative MR analysis of the entorhinal cortex and hippocampus in diagnosing Alzheimer disease, AJNR Am J Neuroradiol 20 (1999), 139-144.

[53] K. Juottonen, M. Lehtovirta, S. Helisalmi, P.J. Riekkinen, Sr. and $\mathrm{H}$. Soininen, Major decrease in the volume of the entorhinal cortex in patients with Alzheimer's disease carrying the apolipoprotein E epsilon4 allele, J Neurol Neurosurg Psychiatry $\mathbf{6 5}$ (1998), 322-327.

[54] G. Karas, J. Sluimer, R. Goekoop, W. van der Flier, S.A. Rombouts, H. Vrenken, P. Scheltens, N. Fox and F. Barkhof, Amnestic mild cognitive impairment: structural MR imaging findings predictive of conversion to Alzheimer disease, AJNR Am J Neuroradiol 29 (2008), 944-949.

[55] G.B. Karas, E.J. Burton, S.A. Rombouts, R.A. van Schijndel, J.T. O'Brien, P. Scheltens, I.G. McKeith, D. Williams, C. Ballard and F. Barkhof, A comprehensive study of gray matter loss in patients with Alzheimer's disease using optimized voxel-based morphometry, NeuroImage 18 (2003), 895-907.

[56] G.B. Karas, P. Scheltens, S.A. Rombouts, P.J. Visser, R.A. van Schijndel, N. C. Fox and F. Barkhof, Global and local gray matter loss in mild cognitive impairment and Alzheimer's disease, Neurolmage 23 (2004), 708-716.

[57] R.J. Killiany, T. Gomez-Isla, M. Moss, R. Kikinis, T. Sandor, F. Jolesz, R. Tanzi, K. Jones, B.T. Hyman and M.S. Albert, Use of structural magnetic resonance imaging to predict who will get Alzheimer's disease, Ann Neurol 47 (2000), 430-439.

[58] R.J. Killiany, B.T. Hyman, T. Gomez-Isla, M.B. Moss, R. Kikinis, F. Jolesz, R. Tanzi, K. Jones and M.S. Albert, MRI measures of entorhinal cortex vs hippocampus in preclinical AD, Neurology 58 (2002), 1188-1196.

[59] R.J. Killiany, M.B. Moss, M.S. Albert, T. Sandor, J. Tieman and F. Jolesz, Temporal lobe regions on magnetic resonance imaging identify patients with early Alzheimer's disease, Arch Neurol 50 (1993), 949-954.

[60] W.R. Markesbery, F.A. Schmitt, R.J. Kryscio, D.G. Davis, C.D. Smith and D.R. Wekstein, Neuropathologic substrate of mild cognitive impairment, Arch Neurol 63 (2006), 38-46.

[61] L.K. McEvoy, C. Fennema-Notestine, J.C. Roddey, D.J. Hagler, Jr., D. Holland, D.S. Karow, C.J. Pung, J.B. Brewer and A.M. Dale, Alzheimer Disease: Quantitative Structural Neuroimaging for Detection and Prediction of Clinical and Structural Changes in Mild Cognitive Impairment, Radiology 251 (2009), 195-205.

[62] C. Misra, Y. Fan and C. Davatzikos, Baseline and longitudinal patterns of brain atrophy in MCI patients, and their use in prediction of short-term conversion to AD: results from ADNI, NeuroImage 44 (2009), 1415-1422. 
[63] E.C. Mormino, J.T. Kluth, C. M. Madison, G.D. Rabinovici, S.L. Baker, B.L. Miller, R.A. Koeppe, C.A. Mathis, M.W. Weiner and W.J. Jagust, Episodic memory loss is related to hippocampal-mediated \{beta\}-amyloid deposition in elderly subjects, Brain (2008).

[64] J.C. Morris, M. Storandt, J.P. Miller, D.W. McKeel, J.L. Price, E.H. Rubin and L. Berg, Mild cognitive impairment represents early-stage Alzheimer disease, Arch Neurol 58 (2001), 397405.

[65] S.G. Mueller, M.W. Weiner, L.J. Thal, R.C. Petersen, C. Jack, W. Jagust, J.Q. Trojanowski, A.W. Toga and L. Beckett, The Alzheimer's disease neuroimaging initiative, Neuroimaging Clin N Am 15 (2005), 869-877, xi-xii.

[66] S.G. Mueller, M.W. Weiner, L.J. Thal, R.C. Petersen, C.R. Jack, W. Jagust, J.Q. Trojanowski, A.W. Toga and L. Beckett, Ways toward an early diagnosis in Alzheimer's disease: The Alzheimer's Disease Neuroimaging Initiative (ADNI), Alzheimers Dement 1 (2005), 55-66.

[67] O. Pedraza, D. Bowers and R. Gilmore, Asymmetry of the hippocampus and amygdala in MRI volumetric measurements of normal adults, J Int Neuropsychol Soc 10 (2004), 664-678.

[68] R.C. Petersen, Mild cognitive impairment as a diagnostic entity, J Intern Med 256 (2004), 183-194.

[69] R.C. Petersen and D. Bennett, Mild cognitive impairment: is it Alzheimer's disease or not? J Alzheimers Dis 7 (2005), 241-245; discussion 255-262.

[70] R.C. Petersen, J.E. Parisi, D.W. Dickson, K.A. Johnson, D.S. Knopman, B.F. Boeve, G.A. Jicha, R.J. Ivnik, G.E. Smith, E.G. Tangalos et al., Neuropathologic features of amnestic mild cognitive impairment, Arch Neurol 63 (2006), 665-672.

[71] A. Ramani, J.H. Jensen and J.A. Helpern, Quantitative MR imaging in Alzheimer disease, Radiology 241 (2006), 26-44.

[72] R.I. Scahill, J.M. Schott, J.M. Stevens, M.N. Rossor and N.C. Fox, Mapping the evolution of regional atrophy in Alzheimer's disease: unbiased analysis of fluid-registered serial MRI, Proc Natl Acad Sci U S A 99 (2002), 4703-4707.

[73] P. Scheltens, N. Fox, F. Barkhof and C. De Carli, Structural magnetic resonance imaging in the practical assessment of dementia: beyond exclusion, Lancet Neurol 1 (2002), 13-21.

[74] N. Schuff, N. Woerner, L. Boreta, T. Kornfield, L.M. Shaw, J.Q. Trojanowski, P.M. Thompson, C.R. Jack, Jr. and M.W. Weiner, MRI of hippocampal volume loss in early Alzheimer's disease in relation to ApoE genotype and biomarkers, Brain (2009).

[75] S.W. Seo, K. Im, J.M. Lee, Y.H. Kim, S.T. Kim, S.Y. Kim, D.W. Yang, S.I. Kim, Y.S. Cho and D.L. Na, Cortical thickness in single- versus multiple-domain amnestic mild cognitive impairment, NeuroImage 36 (2007), 289-297.

[76] V. Singh, H. Chertkow, J.P. Lerch, A.C. Evans, A.E. Dorr and N.J. Kabani, Spatial patterns of cortical thinning in mild cognitive impairment and Alzheimer's disease, Brain 129 (2006), 2885-2893.

[77] A.D. Smith, Imaging the progression of Alzheimer pathology through the brain, Proc Natl Acad Sci U S A 99 (2002), 41354137.

[78] H. Soininen, K. Partanen, A. Pitkanen, M. Hallikainen, T. Hanninen, S. Helisalmi, A. Mannermaa, M. Ryynanen, K. Koivisto and P. Riekkinen, Sr., Decreased hippocampal volume asymmetry on MRIs in nondemented elderly subjects carrying the apolipoprotein E epsilon 4 allele, Neurology 45 (1995), 391-392.

[79] T.R. Stoub, M. Bulgakova, S. Leurgans, D.A. Bennett, D. Fleischman, D.A. Turner and L. deToledo-Morrell, MRI predictors of risk of incident Alzheimer disease: a longitudinal study, Neurology 64 (2005), 1520-1524.

[80] S.J. Teipel, J.C. Pruessner, F. Faltraco, C. Born, M. RochaUnold, A. Evans, H.J. Moller and H. Hampel, Comprehensive dissection of the medial temporal lobe in AD: measurement of hippocampus, amygdala, entorhinal, perirhinal and parahippocampal cortices using MRI, J Neurol 253 (2006), 794-800.

[81] C. Testa, M.P. Laakso, F. Sabattoli, R. Rossi, A. Beltramello, H. Soininen and G.B. Frisoni, A comparison between the accuracy of voxel-based morphometry and hippocampal volumetry in Alzheimer's disease, J Magn Reson Imaging 19 (2004), 274-282.

[82] L.J. Thal, K. Kantarci, E.M. Reiman, W.E. Klunk, M.W. Weiner, H. Zetterberg, D. Galasko, D. Pratico, S. Griffin, D. Schenk et al., The role of biomarkers in clinical trials for Alzheimer disease, Alzheimer Dis Assoc Disord 20 (2006), 6-15.

[83] P.M. Thompson, K.M. Hayashi, G. de Zubicaray, A.L. Janke, S.E. Rose, J. Semple, D. Herman, M.S. Hong, S.S. Dittmer, D.M. Doddrell et al., Dynamics of gray matter loss in Alzheimer's disease, J Neurosci 23 (2003), 994-1005.

[84] P.M. Thompson, K.M. Hayashi, R.A. Dutton, M.C. Chiang, A.D. Leow, E.R. Sowell, G. De Zubicaray, J.T. Becker, O.L. Lopez, H.J. Aizenstein et al., Tracking Alzheimer's disease, Ann N Y Acad Sci 1097 (2007), 183-214.

[85] L.A. van de Pol, A. Hensel, F. Barkhof, H.J. Gertz, P. Scheltens and W.M. van der Flier, Hippocampal atrophy in Alzheimer disease: age matters, Neurology 66 (2006), 236-238.

[86] J.L. Whitwell, S.A. Przybelski, S.D. Weigand, D.S. Knopman, B.F. Boeve, R.C. Petersen and C.R. Jack, Jr., 3D maps from multiple MRI illustrate changing atrophy patterns as subjects progress from mild cognitive impairment to Alzheimer's disease, Brain 130 (2007), 1777-1786.

[87] J.L. Whitwell, M.M. Shiung, S.A. Przybelski, S.D. Weigand, D.S. Knopman, B.F. Boeve, R.C. Petersen and C.R. Jack, Jr., MRI patterns of atrophy associated with progression to AD in amnestic mild cognitive impairment, Neurology 70 (2008), 512-520.

[88] Y. Xu, C.R. Jack, Jr., P.C. O’Brien, E. Kokmen, G.E. Smith, R.J. Ivnik, B.F. Boeve, R.G. Tangalos and R.C. Petersen, Usefulness of MRI measures of entorhinal cortex versus hippocampus in AD, Neurology 54 (2000), 1760-1767. 


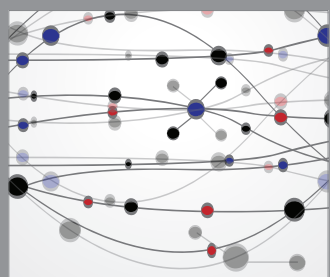

The Scientific World Journal
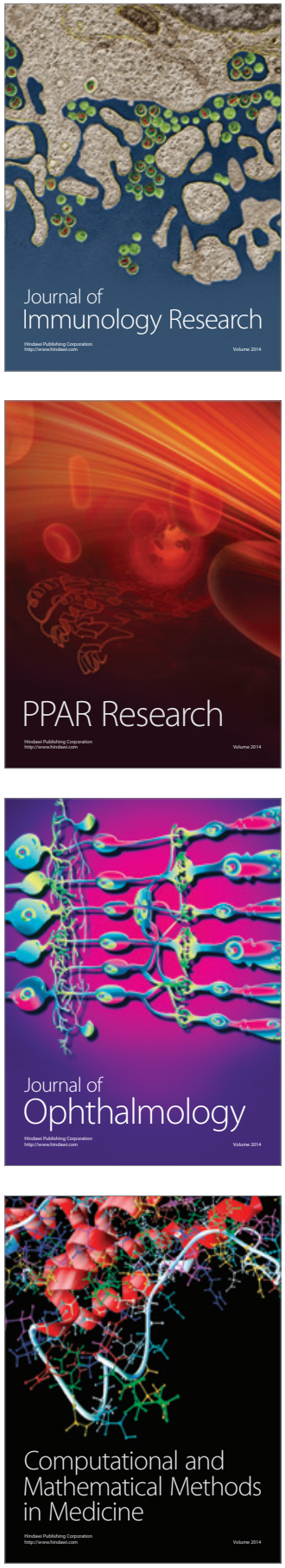

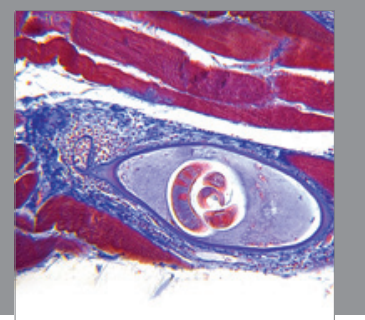

Gastroenterology

Research and Practice
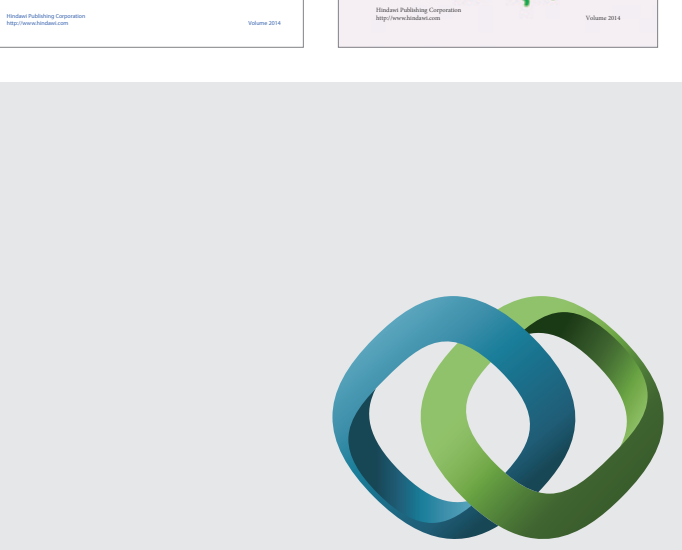

\section{Hindawi}

Submit your manuscripts at

http://www.hindawi.com
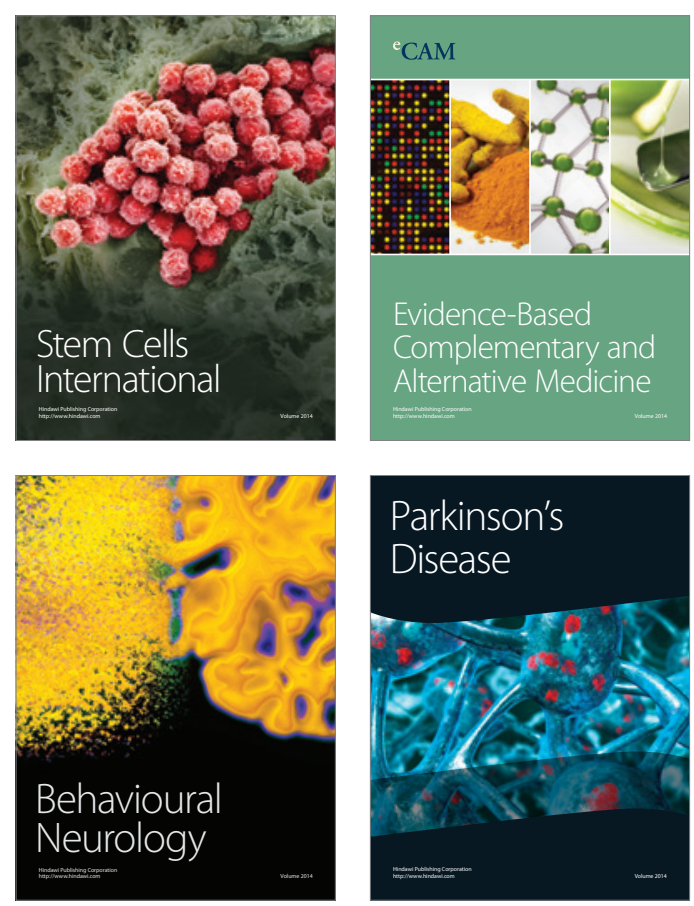

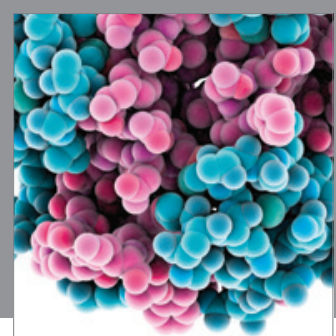

Journal of
Diabetes Research

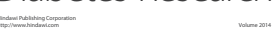

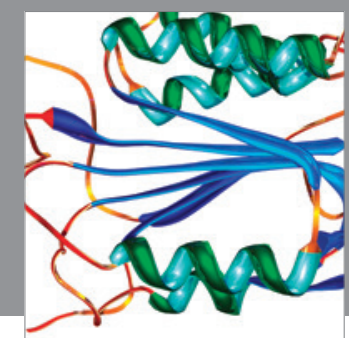

Disease Markers
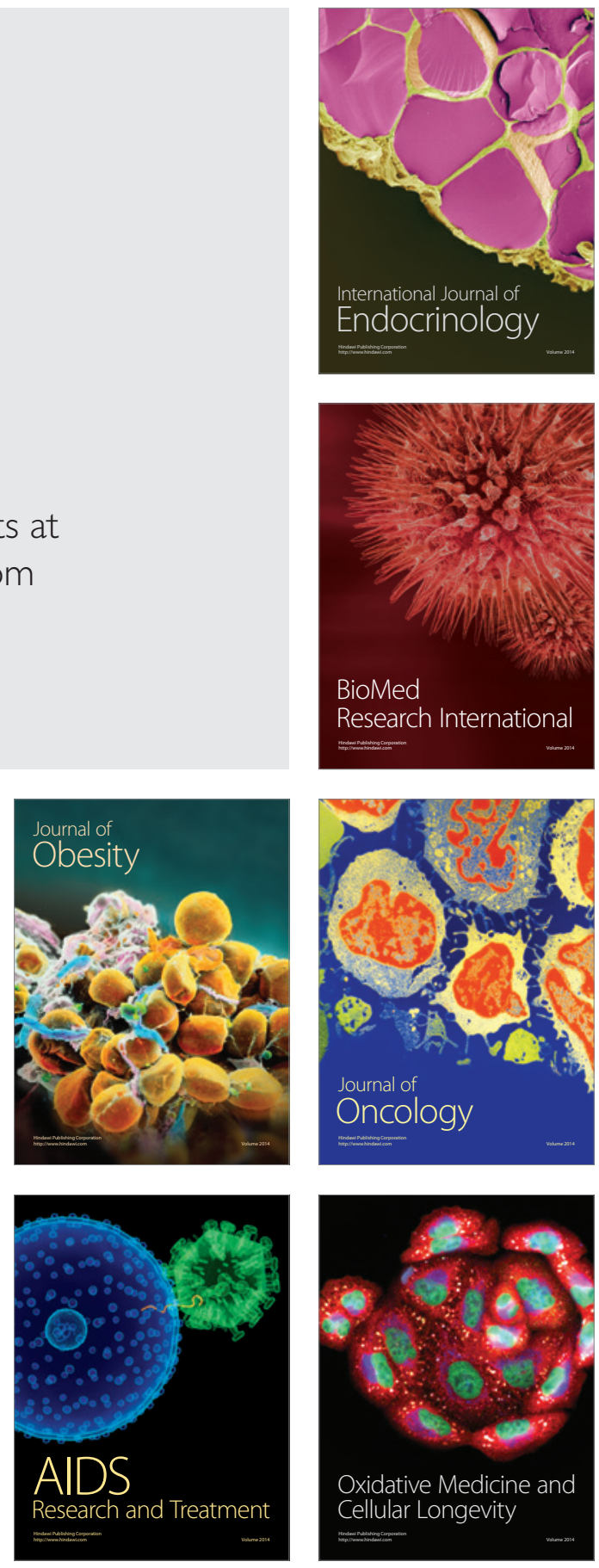